\title{
Population Genetic Structure of Phytophthora cinnamomi Associated with Avocado in California and the Discovery of a Potentially Recent Introduction of a New Clonal Lineage
}

\author{
D. Pagliaccia, E. Pond, B. McKee, and G. W. Douhan
}

Department of Plant Pathology and Microbiology, University of California, Riverside 92521.

Accepted for publication 17 September 2012.

\begin{abstract}
Pagliaccia, D., Pond, E., McKee, B., and Douhan, G. W. 2013. Population genetic structure of Phytophthora cinnamomi associated with avocado in California and the discovery of a potentially recent introduction of a new clonal lineage. Phytopathology 103:91-97.

Phytophthora root rot (PRR) of avocado (Persea americana), caused by Phytophthora cinnamomi, is the most serious disease of avocado worldwide. Previous studies have determined that this pathogen exhibits a primarily clonal reproductive mode but no population level studies have been conducted in the avocado-growing regions of California. Therefore, we used amplified fragment length polymorphism based on 22 polymorphic loci and mating type to investigate pathogen diversity from 138 isolates collected in 2009 to 2010 from 15 groves from the Northern and Southern avocado-growing regions. Additional isolates collected from

avocado from 1966 to 2007 as well as isolates from other countries and hosts were also used for comparative purposes. Two distinct clades of A2 mating-type isolates from avocado were found based on neighbor joining analysis; one clade contained both newer and older collections from Northern and Southern California, whereas the other clade only contained isolates collected in 2009 and 2010 from Southern California. A third clade was also found that only contained A1 isolates from various hosts. Within the California population, a total of 16 genotypes were found with only one to four genotypes identified from any one location. The results indicate significant population structure in the California avocado $P$. cinnamomi population, low genotypic diversity consistent with asexual reproduction, potential evidence for the movement of clonal genotypes between the two growing regions, and a potential introduction of a new clonal lineage into Southern California.
\end{abstract}

Phytophthora cinnamomi Rand is a globally distributed soilborne pathogen that can infect over 3,000 plant species and is a significant problem in forestry, agricultural, and horticultural industries (10). P. cinnamomi is the causal agent of Phytophthora root rot (PRR) of avocado (Persea americana Miller) and is the most serious disease of avocado worldwide (29). The disease has actually eliminated commercial production in many areas in Latin America and is the major limiting factor of production in Australia, South Africa, and California (19). The pathogen was first identified as causing PRR in avocado in Puerto Rico in 1928 and was eventually determined in 1942 to be responsible for a general decline of avocado trees in California that had been reported since the late 1920s and 1930s $(23,26)$. In California, PRR of avocado remains a significant disease problem and has been estimated to affect 60 to $75 \%$ of groves with losses of up to $\$ 40$ million occurring annually (3).

P. cinnamomi is a heterothallic oomycete with known A1 and A2 mating types $(9,21)$. However, the role that oospores play in the epidemiology of various diseases caused by $P$. cinnamomi is not fully understood and most population studies have found that one mating type normally dominates and it is generally thought that the pathogen population structure is predominately clonal (10). Within the avocado- $P$ cinnamomi system, Mircetich and Zentmyer (14) demonstrated that oospores can develop in naturally infested soil and roots but it is not known if oospore formation results in increased genotypic diversity among $P$. cinnamomi populations due to sexual reproduction or how prominent, if at all, oospores form under normal avocado grove conditions. Moreover,

Corresponding author: G. W. Douhan; E-mail address: gdouhan@ucr.edu

http://dx.doi.org/10.1094/PHYTO-01-12-0016-R

(C) 2013 The American Phytopathological Society isolates from avocado predominately belong to the A2 mating type which would limit the chances of sexual reproduction to occur in the field (28) but P. cinnamomi isolates from avocado have also been shown to be homothallic under certain conditions (27). This suggests that oospores could potentially serve as survival structures. However, if they did form under natural conditions, they most likely would be the result of self-fertilization that would likely not result in significant increased genotypic diversity.

It has been hypothesized that the center of origin of $P$. cinnamomi is Papua New Guinea because the greatest amount of genotypic diversity has been found in this area and the fact that many plant species are not susceptible to the pathogen $(12,17,22)$. In contrast, significantly less diversity has been found in Australia and South Africa $(7,12)$ which has led to the speculation that founder populations have spread from Papa New Guinea to these parts of the world, where significant epidemics have occurred, presumably due to $P$. cinnamomi coming into contact with nonnative hosts. For example, the Jarrah forest in Southwestern Australia covers about 4.5 million ha and P. cinnamomi has caused severe forest decline (dieback) in this region because native plants have not evolved with the pathogen and many are highly susceptible to infection (1).

No studies have focused specifically on the genetic structure of $P$. cinnamomi in California for any host and only a few studies have included isolates associated with avocado in general (12, $15,17,18)$. Therefore, the objectives of this study were to characterize isolates of $P$. cinnamomi collected from both the Northern and Southern avocado-growing regions within California using molecular markers (amplified fragment length polymorphism [AFLP]) and mating type. We specifically wanted to determine overall genotypic diversity and to test for population structure based on growing region to make inferences on potential pathogen movement, gene-genotype flow, and to determine if unique 
genotypes or populations exist that could potentially influence the release of avocado rootstocks that are actively being developed for tolerance to PRR of avocado.

\section{MATERIALS AND METHODS}

Isolate collection. Avocado groves exhibiting symptoms of PRR were sampled in 2009 and 2010 to collect P. cinnamomi isolates from areas of the Northern (Santa Barbara Co., Ventura Co.) and Southern (Riverside Co., San Diego Co.) avocado-growing regions in California. A total of 15 locations were sampled that were separated by $0.5 \mathrm{~km}$ to approximately $300 \mathrm{~km}$; seven locations from the Northern avocado-growing region (N1 to N7) and eight locations from the Southern avocado-growing region (S1 to S8) (Fig. 1, Table 1). To isolate the pathogen from the sampled groves, up to 10 trees were sampled that were distributed throughout approximately 200 to $2,000 \mathrm{~m}^{2}$ per grove. Preliminary sampling and analysis of the genotypic diversity of P. cinnamomi in California demonstrated that the population was highly clonal (G. W. Douhan, unpublished data). Therefore, our sampling strategy was to sample as many locations as feasible and only sample up to 10 trees per location rather than conduct a more intensive sampling at fewer locations. A single soil sample per tree was collected using a shovel from beneath the canopy within the sprinkler line. Up to ten $1.0-\mathrm{cm}$ root pieces from each sample were rinsed with water, dipped into $75 \% \mathrm{EtOH}$ to surface sterilize, and plated into PARPH agar (1/2 clarified V8 juice agar containing $0.02 \mathrm{~g}$ of pimaricin, $0.25 \mathrm{~g}$ of ampicillin, $0.01 \mathrm{~g}$ of rifampicin, $0.100 \mathrm{~g}$ of PCNB [Terachlor], and $0.075 \mathrm{~g}$ of hymexazole per liter $1 / 2$ clarified $V 8$ juice agar). Five roots were imbedded in the agar on each plate and two plates were made per soil sample. Resulting P. cinnamomi colonies (1 to 6) growing from the roots were transferred several times to clean PARPH plates and eventually hyphal tipped to obtain pure cultures. Sample identification code was based on a three number system; the first number designated the sampled grove, followed by the sampled tree, followed by the specific isolate. For extraction of DNA, mycelium was aseptically scraped from the plates and transferred to $2 \times$ cetyltrimethylammonium bromide (CTAB) solution and kept frozen for later use. DNA was isolated with a modified phenol-chloroform extraction procedure (11). The quality and quantity was evaluated in $0.8 \%$ agarose gels stained with SYBR Green I (Molecular Probes, Eugene, OR).

Additional isolates of $P$. cinnamomi obtained from California avocado groves were also used in this study. These 12 cultures had been kept in long-term storage at $10^{\circ} \mathrm{C}$ on slant cultures from a culture collection of isolates (M253 to M386) sampled between 1993 and 2007 (Table 1). DNA from known A1 and A2 isolates of P. cinnamomi (A1495 to A1581) from avocado, soil, and other hosts originally collected from California and elsewhere were also obtained from the World Phytophthora and Oomycete Genetic Resource Collection (WOC) located at the Department of Plant Pathology and Microbiology at the University of CaliforniaRiverside (Table 1).

Determination of mating type. Mating type was determined for all new P. cinnamomi isolates recovered for this study as well as for some of the isolates from our culture collection where mating type had not been determined previously. Mating type for the isolates from the WOC were previously determined. Each isolate was crossed with (i) a known A1 mating-type isolate (M416 from Camellia japonica provide by M. Garbelotto, UC Berkeley), (ii) a known A2 isolate (M295 avocado), and (iii) crossed with itself. A mycelial plug from each individual was transferred to one side of a clarified V8 juice agar plate while a mycelial plug from the cross was transferred to the other side of the plate. After 1 week incubation in the dark, each plate was observed microscopically using a NIKON SMZ-800 stereomicroscope (Nikon Instruments Inc.) to observe whether or not oospores formed.

AFLP. The method of Vos et al. (25) was used to develop the initial restriction-ligation products used for AFLP but fluorescently labeled primers were used to resolve the markers via a capillary sequencer as described below. A total of 12 randomly

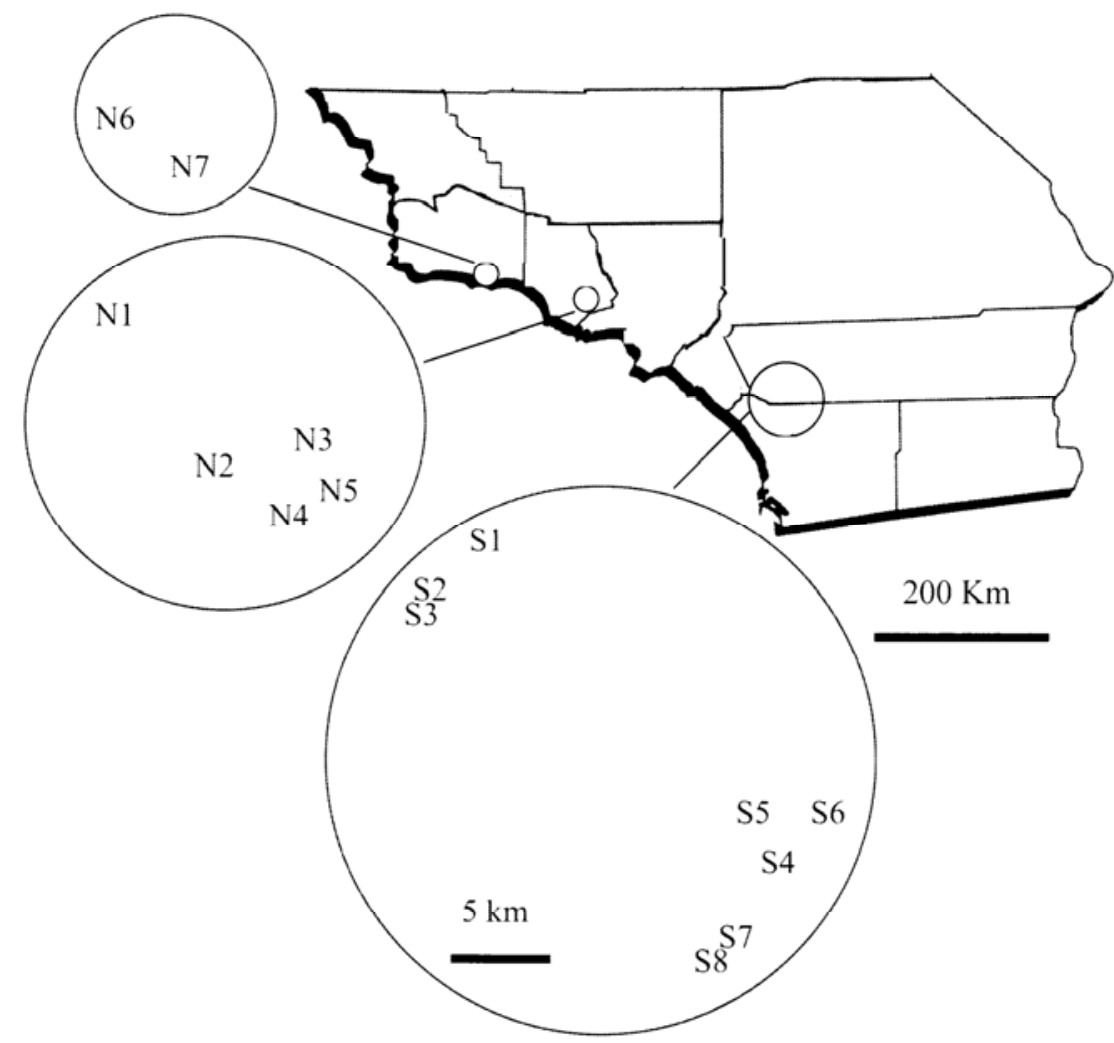

Fig. 1. Geographical map showing the locations where groves were sampled for Phytophthora cinnamomi from the Northern (N) and Southern (S) avocadogrowing regions of California. 
chosen primer combinations with the addition of two or three selective nucleotides at the $3^{\prime}$ end were used to screen a subset of eight isolates and four primer combinations were chosen for the final analyses which resolved the fragment patterns well under the experimental conditions used. The four chosen primer combinations had the sequences for the EcoRI $(E+0)$ and MseI $(M+0)$ adapters followed by the addition of FAM ECORI +GC/ MSEI + AT, FAM ECORI + GC/MSEI + GG, HEX ECORI +GCT/MSEI + $\mathrm{AG}$ and HEX ECORI + TA/MSEI + CG at the 3 prime ends. Each $20-\mu$ reaction contained $1 \times$ polymerase chain reaction (PCR) buffer (Invitrogen, Carlsbad, CA), $2.5 \mathrm{mM} \mathrm{MgCl} 2,2.5 \mathrm{mM}$ each dNTP (Invitrogen), $0.375 \mu \mathrm{M}$ of each primer, $0.5 \mathrm{U}$ of Taq polymerase (Invitrogen), and $10 \mu \mathrm{l}$ of template of the restrictionligation dilution. Nine isolates were used as controls by conducting independent DNA extractions, restriction-ligation reactions, and PCR amplifications. Thermocycling conditions consisted of an initial hold at $72^{\circ} \mathrm{C}$ and $94^{\circ} \mathrm{C}$ for 1 and $4 \mathrm{~min}$, respectively, followed by 44 cycles with an annealing temperature of $65^{\circ} \mathrm{C}$ for $30 \mathrm{~s}$ and an extension temperature of $72^{\circ} \mathrm{C}$ for 1 min with the annealing temperature reduced by $1^{\circ} \mathrm{C}$ for the first nine cycles. All amplifications were performed in a MyCycler (Bio-Rad Laboratories).

The labeled FAM and HEX fragments were detected using an ABI 3100 16-capillary instrument at the CORE Instrumentation Facility at UC-Riverside. Genographer 2.0 fragment analysis software was used to score the data by setting the intensity settings between 1 and 5 and manually scoring the presence or absence of clearly visible polymorphic bands above 100 bp by checking chromatograms as well as an electronic image of the gel. Putative alleles at each polymorphic AFLP locus were scored using a binary code $(1,0)$ corresponding to positive and null alleles, respectively. For the selection of AFLP markers, only polymorphic loci that were reproducible and easily identifiable were chosen.

Data analysis. Cluster analyses on genetic distances, using neighbor joining $(\mathrm{NJ})$ and the mean character difference option and unweighted pair-group method with arithmetic averaging (UPMGA), were conducted using PAUP* (version 4.0 beta 10) (24). Confidence in tree topology was examined using the nonparametric bootstrap with 1,000 bootstrapped replicates using PAUP. Two data sets were analyzed; one data set with all A1 and A2 isolates used in this study $(n=161)$ and one containing only A2 isolates $(n=158)$. Estimates of genotypic diversity were not calculated since sample sizes in some locations were very low and also because only one to four genotypes were recovered from any one location (see Results).

Population structure and assignment of isolates to populations was also inferred from the AFLP multilocus genotypes using a Bayesian model-based clustering method implemented in the STRUCTURE software package (20). For this analysis, only isolates that were collected from avocado in 2009 and $2010(n=$ 138) were used. The model evaluated allowed correlated allele frequencies and individuals to have mixed ancestry. Analyses

TABLE 1. Isolate information for Phytophthora cinnamomi samples used in this study

\begin{tabular}{|c|c|c|c|c|c|c|c|}
\hline Isolate/population & No. of isolates & Country/state & County & Collector & Host/origin & Date & Mating type \\
\hline N1 & 2 & $\mathrm{CA}$ & Ventura & E. Pond & Avocado & 2010 & $\mathrm{~A} 2$ \\
\hline $\mathrm{N} 2$ & 2 & $\mathrm{CA}$ & Ventura & E. Pond & Avocado & 2009 & A2 \\
\hline N3 & 15 & $\mathrm{CA}$ & Ventura & E. Pond & Avocado & $2009 / 10$ & $\mathrm{~A} 2$ \\
\hline N4 & 16 & $\mathrm{CA}$ & Ventura & E. Pond & Avocado & $2009 / 10$ & $\mathrm{~A} 2$ \\
\hline N5 & 1 & $\mathrm{CA}$ & Ventura & E. Pond & Avocado & 2010 & $\mathrm{~A} 2$ \\
\hline N6 & 13 & $\mathrm{CA}$ & Santa Barbara & E. Pond & Avocado & 2010 & $\mathrm{~A} 2$ \\
\hline N7 & 8 & $\mathrm{CA}$ & Santa Barbara & E. Pond & Avocado & 2010 & A2 \\
\hline $\mathrm{S} 1$ & 18 & $\mathrm{CA}$ & Riverside & E. Pond & Avocado & 2009 & A2 \\
\hline $\mathrm{S} 2$ & 13 & $\mathrm{CA}$ & Riverside & E. Pond & Avocado & 2009 & A2 \\
\hline $\mathrm{S} 3$ & 14 & $\mathrm{CA}$ & Riverside & E. Pond & Avocado & 2009 & $\mathrm{~A} 2$ \\
\hline S4 & 5 & $\mathrm{CA}$ & San Diego & E. Pond & Avocado & 2009 & A2 \\
\hline S5 & 6 & $\mathrm{CA}$ & San Diego & E. Pond & Avocado & 2009 & $\mathrm{~A} 2$ \\
\hline S6 & 11 & $\mathrm{CA}$ & San Diego & E. Pond & Avocado & 2009 & A2 \\
\hline S7 & 6 & $\mathrm{CA}$ & San Diego & E. Pond & Avocado & 2009 & $\mathrm{~A} 2$ \\
\hline S8 & 8 & $\mathrm{CA}$ & San Diego & E. Pond & Avocado & 2009 & $\mathrm{~A} 2$ \\
\hline M253 & 1 & $\mathrm{CA}$ & San Diego & G. King & Avocado & 1993 & $\mathrm{~A} 2$ \\
\hline M254 & 1 & $\mathrm{CA}$ & San Diego & G. King & Avocado & 1993 & $\mathrm{~A} 2$ \\
\hline M262 & 1 & $\mathrm{CA}$ & San Diego & $\mathrm{ND}^{\mathrm{b}}$ & Avocado & 1994 & A2 \\
\hline M280 & 1 & $\mathrm{CA}$ & Ventura & ND & Avocado & 1995 & A2 \\
\hline M281 & 1 & $\mathrm{CA}$ & Ventura & ND & Avocado & 1995 & A2 \\
\hline M282 & 1 & $\mathrm{CA}$ & Ventura & ND & Avocado & 1995 & A2 \\
\hline M293 & 1 & $\mathrm{CA}$ & Ventura & ND & Avocado & 1996 & A2 \\
\hline M295 & 1 & $\mathrm{CA}$ & Ventura & J. Downer & Avocado & 1996 & A2 \\
\hline M381 & 1 & $\mathrm{CA}$ & Riverside & T. Shea & Avocado & 2006 & $\mathrm{~A} 2$ \\
\hline M382 & 1 & $\mathrm{CA}$ & San Diego & T. Shea & Avocado & 2007 & A2 \\
\hline M385 & 1 & $\mathrm{CA}$ & Santa Barbara & ND & Avocado & 2007 & A2 \\
\hline M386 & 1 & $\mathrm{CA}$ & Santa Barbara & ND & Avocado & 2007 & $\mathrm{~A} 2$ \\
\hline A1495 & 1 & $\mathrm{CA}$ & Los Angeles & G. Zentmyer & Pine & 1975 & A 2 \\
\hline A1496 & 1 & $\mathrm{CA}$ & Riverside & G. Zentmyer & Azalea & 1976 & $\mathrm{~A} 2$ \\
\hline A1498 & 1 & FL & ND & R. Ploetz & Avocado & 1989 & $\mathrm{~A} 2$ \\
\hline A1574 & 1 & $\mathrm{CA}$ & Riverside & G. Zentmyer & Rhododendron & 1976 & $\mathrm{~A} 2$ \\
\hline A1494 & 1 & China & ND & P. Tsao & Cameallia & 1986 & A1 \\
\hline A1497 & 1 & $\mathrm{CA}$ & Santa Barbara & G. Zentmyer & Avocado & 1970 & A1 \\
\hline A1575 & 1 & Madagascar & ND & ND & Avocado & 1966 & A1 \\
\hline A1576 & 1 & Papa New Guinea & ND & ND & Soil & ND & A1 \\
\hline A1579 & 1 & China & ND & ND & Rhododendron & 1989 & A1 \\
\hline A1580 & 1 & South Africa & ND & ND & Grape & ND & A1 \\
\hline A1581 & 1 & Taiwan & ND & ND & Pineapple & 1989 & A1 \\
\hline \multicolumn{8}{|l|}{ Total number } \\
\hline
\end{tabular}

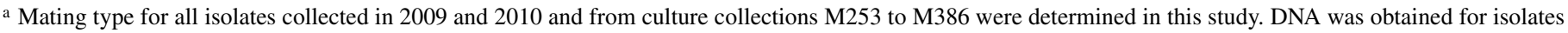
A1495 to A1581 and the mating type information was obtained from the World Phytophthora Genetic Resource Collections database.

b ND = no data available. 
consisted of $10^{5}$ burn-in replicates and a run length of $10^{6}$ replicates. Ln likelihood values and posterior probabilities were estimated assuming one to six population clusters $(K=1,2 \ldots 6)$ and each analysis was conducted independently 10 times. The output files for all of the analyses were placed in a .ZIP file and uploaded to the STRUCTURE HAVERSTER website (http://taylor0.biology.ucla.edu/struct_harvest/) which uses the Evanno et al. (8) method to determine the most probable cluster ( $K$ value), i.e., the most probable number of populations.

\section{RESULTS}

Mating type. All isolates collected from the 15 locations were determined to belong to the A2 mating type. All of our older $P$. cinnamomi cultures (M253 to M386) originally collected from avocado were also found to belong to the A2 mating type (Table 1). Four additional A2 isolates (A1495 to A1574) from pine, azalea, avocado, and rhododendron were also used in some of the analyses as described below as well as seven A1 isolates obtained from the WOP which were originally collected from various hosts and origins (Table 1).

AFLP analysis. A total of 138 isolates of $P$. cinnamomi were obtained from the 15 sampled locations with sample sizes ranging from $n=1$ to $n=18$ (Table 2). The nine isolates used in independent extractions, restriction-ligations, and PCR amplifications for controls produced identical banding patterns as their original cultures. Therefore, only a single representative of each control was used in the analysis along with the rest of the isolates. The AFLP fingerprints from all four primer combinations resulted in 133 bands that could be unambiguously scored, of which 22 (16.54\%) were polymorphic among P. cinnamomi isolates.

Based on the analysis of 22 polymorphic AFLP markers, a total of 16 unique genotypes were found from the 15 sampled locations and the number of genotypes found per population ranged from 1 to 4 (Table 2). When comparing genotypes across the populations, genotype 1 was dominant and was found 45 times and was sampled from 10 locations (Table 2). All of the other genotypes were only isolated from one to three locations and the number of times these other genotypes were found ranged from 1 to 24 times (Table 2).

NJ and UPGMA analyses. When all of the 161 isolates were used in the analysis, three distinct clades were found; one clade containing only A1 isolates and the other two clades containing A2 isolates (data not shown). Both NJ and UPGMA produced similar trees; therefore, only an NJ tree is shown based on A2 isolates since all California avocado isolates used in this study were the A2 mating type except for one isolate (A1497) collected in 1970 which is the only known A1 mating-type isolate ever reported from California.

Based on the $\mathrm{NJ}$ analysis, two distinct A2 clades were found that we will refer to as A2 clade I and A2 clade II, which were supported by $98 \%$ bootstrap support (Fig. 2). Additional bootstrap support was also found within both main clades but most of the support values were minimal (Fig. 2). A total of 10 genotypes were detected in the A2 clade I group associated with avocado and two additional isolates associated with pine and rhododendron were also identical to two of the genotypes (Table 1; Fig. 2). Four of the ten genotypes were found in avocado groves from both the Northern and Southern California collecting locations. An 11th genotype was also found which was originally isolated from azalea and two of the isolates within the A2 clade I group were previously identified as belonging to the CINN4 and CINN5 isozyme genotypes (3) that correspond to the previously identified two A2 clonal lineages of $P$. cinnamomi associated with avocado (7). A total of six genotypes were detected in the A2 clade II group. All of the isolates in this clade were collected only from the Southern California growing region from five out of the eight sampled locations and were only isolated in 2009 and 2010 (Fig. 2).

STRUCTURE analysis. The STRUCTURE analysis strongly supported two clusters or populations based on the highest delta $k$ value of 176.6 at $K=2$. This result also correlated with the two A2 clades identified based on the $\mathrm{NJ}$ analysis.

\section{DISCUSSION}

This is the first study to investigate pathogen diversity of P. cinnamomi in California associated with PRR of avocado. The overall results are consistent with a highly clonal population structure based on the identification of only 16 genotypes out of 138 isolates collected from 15 locations among the Northern and Southern avocado-growing regions. However, the NJ analysis based on genetic distances and the population assignment tests based on genotypic data were both consistent with a split of the isolates into two main clades; one containing only isolates from the Southern California growing region (A2 clade II) and another consisting of isolates from both the Northern and Southern California regions (A2 clade I). The results indicate significant population structure in the California avocado $P$. cinnamomi population, low genotypic diversity and one mating type (A2), which is consistent with asexual reproduction. The results also suggest that some clonal genotypes have been disseminated throughout California since identical genotypes were isolated from the sampled locations within each region and between the two growing regions.

TABLE 2. Occurrence and distribution of multilocus genotypes of Phytophthora cinnamomi sampled in 2009 to 2010 from 15 avocado groves in the Northern (N) and Southern (S) growing regions in California

\begin{tabular}{|c|c|c|c|c|c|c|c|c|c|c|c|c|c|c|c|c|c|c|}
\hline \multirow[b]{2}{*}{ Genotype } & \multirow[b]{2}{*}{ Multilocus data } & \multicolumn{17}{|c|}{ Distribution of genotypes } \\
\hline & & N1 & N2 & N3 & N4 & N5 & N6 & N7 & S1 & S2 & S3 & S4 & S5 & S6 & S7 & S8 & $\begin{array}{l}\text { Number of } \\
\text { times found }\end{array}$ & $\begin{array}{l}\text { Number of popula- } \\
\text { tions observed in }\end{array}$ \\
\hline 1 & 1000011000001100001010 & & 1 & 1 & 10 & 1 & 1 & 8 & 15 & & & 3 & & & 4 & 1 & 45 & 10 \\
\hline 3 & 1000011000001100001011 & & 1 & & & & & & 2 & & & & & & & 2 & 5 & 3 \\
\hline 4 & 1000001000001100001010 & & & & & & & & 1 & & & & & & & & 1 & 1 \\
\hline 5 & 1011011000001100001010 & & & & 4 & & & & & & & & & & & & 4 & 1 \\
\hline 9 & 1000010010001100001110 & & & 7 & & & & & & & & & & & & & 7 & 1 \\
\hline 10 & 1011010010001100001110 & & & & 1 & & & & & & & & & & & & 1 & 1 \\
\hline 11 & 0011010011110011000100 & & & & & & & & & 13 & 11 & & & & & & 24 & 2 \\
\hline 12 & 0011010011110011000101 & & & & & & & & & & 3 & & & & & & 3 & 1 \\
\hline 13 & 0011010011010011000100 & & & & & & & & & & & 2 & 6 & 6 & & & 14 & 3 \\
\hline 14 & 0011010011010011000101 & & & & & & & & & & & & & 3 & & & 3 & 1 \\
\hline
\end{tabular}


The population genetic structure found also suggests that the isolates within the A2 clade II group may be due to a more recent introduction since all of the isolates found in this clade were less diverse than the A2 clade I group, were only collected from Southern California, and were only found from recent isolations. In contrast, isolates from the A2 clade I group were more diverse, consisted of all A2 isolates from older collections, and were also related to the CINN4 and CINN5 isozyme genotypes previously found by Oudemans and Coffey (18). These results are also supported by phylogenetic analysis of multiple mitochondrial loci from all 16 genotypes found in this study (F. Martin and G. W. Douhan, unpublished data). For example, Martin and Coffey (13)

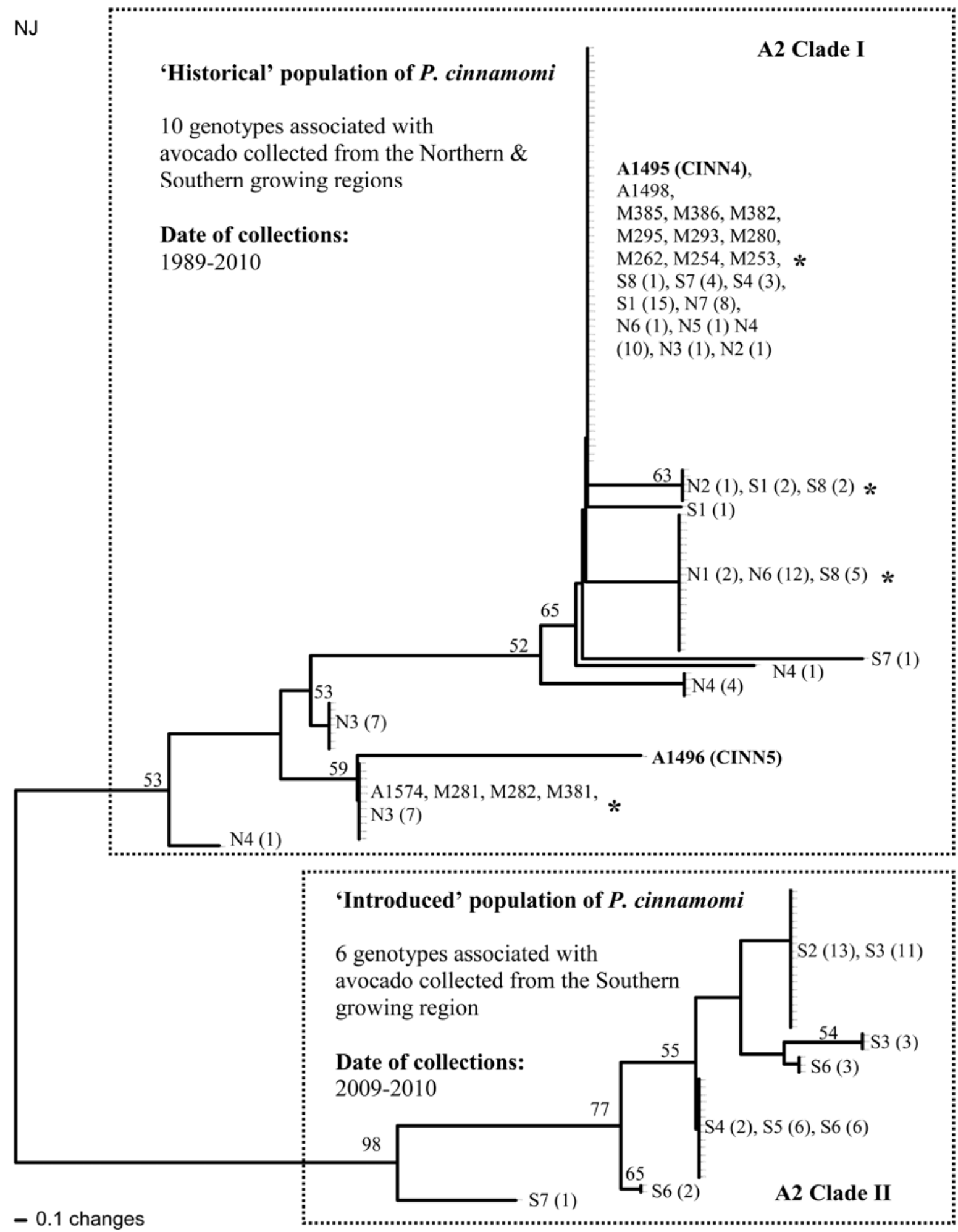

Fig. 2. Neighbor joining analysis of the A2 mating-type Phytophthora cinnamomi isolates used in this study based on 22 amplified fragment length polymorphism markers using PAUP. Bootstrap support of $50 \%$ or more is noted on the tree based on 1,000 replicates of the data. Isolate/population names used are noted for each unique genotype based on the information in Table 1 followed by the actual number of genotypes found in parentheses. Asterisks are noted for genotypes that were found in both the Northern and Southern California avocado-growing regions and isolates that represent the CINN4 and CINN5 isozyme genotypes are also noted (21). Dashed boxes define the putative historic A2 clade I and introduced A2 clade II isolates. 
have recently conducted a multi gene mitochondrial haplotype analysis of a worldwide collection of $P$. cinnamomi isolates. Our preliminary analysis of isolates from the A2 clade II have revealed that these isolates are related to the H1-4 haplotypes found by Martin and Coffey (13) which were collected from P. americana in Puerto Rico, Spain, and South Africa and from Quercus suber in Portugal and from Rhododendron sp. in Spain. Therefore, at this point, it is impossible to know where this putative new introduction came from.

Based on previous population studies, $P$. cinnamomi is thought to consist primarily of three clonal lineages that have been found in Australia, South Africa, and in many areas around the world $(7,10,12)$. The earliest studies were based on isozyme markers that identified only three common multilocus genotypes; two genotypes consisted of isolates with the A2 mating type found in Australia and Papa New Guinea and one genotype consisted of isolates with the A1 mating type from Australia (16,17). These same clonal genotypes, along with some additional genotypes based on additional molecular markers, were later identified from a worldwide collection of $P$. cinnamomi isolates from many different host species. However, all studies found a general lack of genotypic diversity within the species and suggested clonal spread of the pathogen throughout much of the world (18). A later study based on more variable molecular markers, restriction fragment length polymorphisms and randomly amplified polymorphic DNA (RAPD), was also consistent with low genotypic diversity and a lack of evidence for sexual reproduction (13). Dobrowolski et al. (7) investigated populations of $P$. cinnamomi from Southwest Australia using microsatellite markers and found some polymorphisms within the main three clonal lineages identified previously using isozymes but also determined that the population structure was not consistent with a random mating hypothesis based on multilocus gametic disequilibrium analyzes. However, they also found significant diversity among the A1 isolates from Papua New Guinea, similar to the results of Old et al. $(16,17)$, confirming the suggestion that this region may be located within the center of origin of this species.

Only a few studies have included $P$. cinnamomi isolates associated with avocado when determining pathogen diversity. Old et al. (16) using isozymes included 14 isolates; 13 were identified as belonging to the A2 clonal lineage 1, one was from A2 clonal lineage 2, and a single isolate from avocado "soil" was found to be an A1 genotype. Linde et al. (12) included four isolates from South Africa and three isolates from Australia; six genotypes could be resolved based on RAPD markers and all belonged to the A2 mating type. Oudemans and Coffey (18) included the most diverse collection of $P$. cinnamomi isolates from avocado but also characterized the pathogens using low variable isozymes markers. They included 31 avocado isolates sampled from Indonesia, South Africa, Taiwan, Madagascar, Florida, and California. Only five isozyme genotypes were found; three were identified as CINN1 from China and California, CINN6 from Papa New Guinea, and CINN2 from Madagascar, all from single collections, which were identified as belonging to the A1 mating type. The remaining avocado isolates all belonged to the CINN4 or CINN5 genotypes, which were identified as belonging to the A2 mating type and correlated to the previously identified A2 type 1 and A2 type 2 lineages, respectively (7).

In this study, we used isolates that were identified as belonging to the A2 type 1 lineage $(\mathrm{A} 1495=\mathrm{CINN} 4)$ and the A2 type 2 lineage $(\mathrm{A} 1496=\mathrm{CINN} 5)$ but both clustered within the A2 clade I group. We could also resolve 10 clonal genotypes distributed in both the Northern and Southern growing regions within this clade based on the AFLP data. Moreover, we found that all historic isolates that were used in this study from avocado, except for one A1 isolate collected from avocado (A1497), all belonged to the A2 clade I group and likely represents the historical population of $P$. cinnamomi in California. The one A1 isolate from avocado was isolated by the State Department of Agriculture in 1970 from a grove in Santa Barbara and Zentmyer hypothesized that this isolate may have moved into the avocado grove from ornamental landscaping plants that were close to the grove (28). As previously mentioned, no additional A1 isolates have ever been isolated from avocado to the best of our knowledge.

Most studies investigating $P$. cinnamomi diversity associated with PRR are all consistent with overall low genotypic diversity found within this species globally where avocados are cultivated. In contrast, Ochoa-Fuentes et al. (15) have recently investigated $P$. cinnamomi diversity in Mexico using AFLP markers. Based on an analysis of 38 isolates collected from five municipalities within the state of Michoacan, they found that all isolates represented unique genotypes. If correct, the report of high levels of genotypic diversity in Mexico by Ochoa-Fuentes et al. (15) would also be consistent with the possibility that $P$. cinnamomi could also be indigenous to this region. However, the work of Zentmyer suggests that this is not likely. For example, Zentmyer (30) attempted to isolate P. cinnamomi from the roots of native Persea species and related plants from over 400 tree samples from 17 countries in Latin America. P. cinnamomi was never recovered from any of the samples from native and undisturbed areas, even though the moisture and soil conditions were favorable for disease, whereas $P$. cinnamomi was recovered from native species only in areas where susceptible crops were grown nearby.

In contrast, $P$. cinnamomi has readily been isolated from nonsymptomatic native tree species as well as from susceptible crops in other areas of Southeast Asia where the pathogen has been hypothesized to be indigenous $(2,30)$. These additional studies have also reported striking difference between areas where the two mating types were isolated with $\mathrm{A} 1$ isolates obtained from native forests that were very difficult to access while A2 isolates were recovered from areas where frequent exotic contact and plant movement occurred (2,6). Crandall and Gravatt (6) suggested that the pathogen may have been carried with plant material from Asia throughout the Pacific Islands on various trade routes and similarly to Africa, Europe, and to various ports in the Americas. The pathogen may have been introduced into Mexico and some of the other Latin American countries on voyages by Spanish and other explorers from Asia or from the AustralianMalaysian area. Importation of plants from tropical America, including Mexico, occurred commonly beginning in the eighteenth century with Spanish exploration and establishment in the late nineteenth century and the early twentieth centuries of missions in Southern California. There are a number of reports of avocado seedlings and seeds being imported into California, particularly from Mexico $(4,5)$, which could have provided an ingress for the pathogen into Southern California.

Thus, our results are not in agreement with Ochoa-Fuentes et al. (15) work but are consistent with previous observations that the pathogen may have been carried with plant material from Asia on various trade routes to the Americas, Europe, and Africa and is not indigenous to California. Once in the United States, $P$. cinnamomi has probably been disseminated by common methods such as nursery stock, soil movement, man, animals, equipment, and/or water movement. For example, some of the same clonal genotypes were isolated within each region and between regions. $P$. cinnamomi is not capable of such long distance dissemination, especially between the Northern and Southern growing areas, and the most likely explanation for our results would be the movement of these isolates via soil from picking bins. For example, picking bins can be in one growing region one day and several days later they can be shipped to the other and the soil is not routinely cleaned off the bins at the packing houses $(\mathrm{G}$. W. Douhan, personal observation).

Results from this study may have implications for rootstock breeding since two main clades were found among California isolates of P. cinnamomi and only one clade (A2 clade I) contained 
historic isolates associated with avocado that have previously been determined to belong to the known two A2 clonal lineages (7). Isolates from the putatively introduced A2 clade II group were genetically distinct from the A2 clade I group which raises the question as to how pathogenic or virulent these stains are. Moreover, this study also resolved many distinct genotypes within both clades that will allow virulence studies to be determined. With respect to rootstock breeding, this will be valuable information because the initial germplasm disease screening process needs to include isolates that represent the actual diversity of the pathogen population throughout the avocado producing regions of California.

\section{ACKNOWLEDGMENTS}

We thank the California Avocado Commission for funding as well as financial support from the Agricultural Experiment Station, University of California Riverside. We also thank F. Martin, USDA-ARS, for providing preliminary mitochondrial sequence data and M. Coffey, University of California Riverside, for providing DNA from the WOP.

\section{LITERATURE CITED}

1. Anderson, P., Brundrett, M., Grierson, P., and Robinson, R. 2010. Impact of severe forest dieback caused by Phytophthora cinnamomi on macrofungal diversity in the Northern jarrah forest of Western Australia. Forest Ecol. Manag. 259:1033-1040.

2. Arentz, F., and Simpson, I. A. 1986. Distribution of Phytophthora cinnamomi in Papua New Guinea and notes on its origin. Trans. Br. Mycol. Soc. 87:289-295

3. Coffey, M. D. 1992. Phytophthora root rot of avocado. Plant diseases of international importance. Pages 423-444 in: Volume III. Diseases of Fruit Crops. J. Kumar, H. S. Chaube, U. S. Singh, and A. N. Mukhopadhyay, eds. Prentice Hall, Englewood Cliffs, NJ.

4. Condit, I. J. 1917. History of the avocado and its varieties in California with a check list of all named varieties. Annu. Rep. Calif. Avocado Assoc. 105-144.

5. Condit, I. J. 1926. Check list of avocado varieties. Annu. Rep. Calif. Avocado Assoc. 3-20.

6. Crandall, B. S., and Gravatt, G. F. 1967. The distribution of Phytophthora cinnamomi. CEIBA 13:43-53.

7. Dobrowolski, M. P., Tommerup, I. C., Shearer, B. L., and O'Brien, P. A. 2003. Three clonal lineages of Phytophthora cinnamomi in Australia revealed by microsatellites. Phytopathology 93:695-704.

8. Evanno, G., Regnaut, S., and Goudet, J. 2005. Detecting the number of clusters of individuals using the software STRUCTURE: A simulation study. Mol. Ecol. 14:2611-2620.

9. Galindo, A. J., and Zentmyer, G. A. 1964. Mating types in Phytophthora cinnamomi. Phytopathology 54:238-239.

10. Hardham, A. R. 2005. Phytophthora cinnamomi. Mol. Plant Pathol. 6:589-604.

11. Lee, S. B., and Taylor, J. W. 1990. Isolation of DNA from fungal mycelia and single spores. Page 282-287 in: PCR Protocols: A Guide to Methods, Applications. M. A. Innis, D. H. Gelfand, J. J. Sninsky, and T. J. White eds. Academic Press, San Diego.

12. Linde, C., Drenth, A., and Wingfield, M. J. 1999. Gene and genotypic diversity of Phytophthora cinnamomi in South Africa and Australia revealed by DNA polymorphisms. Eur. J. Plant Pathol. 105:667-680.

13. Martin, F. N., and Coffey, M. D. 2012. Mitochondrial haplotype analysis for differentiation of isolates of Phytophthora cinnamomi. Phytopathology 102:229-239.

14. Mircetich, S. M., and Zentmyer, G. A. 1966. Production of oospores and chlamydospores of Phytophthora cinnamomi in roots and soil. Phytopathology 56:1076-1078.

15. Ochoa-Fuentes, Y. M., Martinez-de la Vega, O., Olalde-Portugal, V., Cema-Chavez, E., Landeros-Flores, J., Hemandez-Castillo, F. D., and Flores-Olivas, A. 2007. Genetic variability of Phytophthora cinnamomi Rands in Michoacan, Mexico. Rev. Mex. Fitopatol. 25:161-166.

16. Old, K. M., Dudzinski, M. J., and Bell, J. C. 1988. Isozyme variability in field populations of Phytophthora cinnamomi in Australia. Austr. J. Bot. 36:355-360.

17. Old, K. M., Moran, G. F., and Bell, J. C. 1984. Isozyme variability among isolates of Phytophthora cinnamomi from Australia and Papua New Guinea. Can. J. Bot. 62:2016-2022.

18. Oudemans, P., and Coffey, M. D. 1991. Isozyme comparison within and among worldwide sources of three morphologically distinct species of Phytophthora. Mycol. Res. 95:19-30.

19. Ploetz, R., and Schnell, R. J., and Haynes, J. 2002. Variable response of open-pollinated seedling progeny of avocado to Phytophthora root rot. Phytoparasitica 30:262-268

20. Pritchard, J. K., Stephens, P., and Donnelly, P. 2000. Inference on population structure using multilocus genotypic data. Genetics 155:945959.

21. Savage, E. J., Clayton, C. W., Hunter, J. H., Brenneman, J. A., Laviola, C., and Gallegly, M. E. 1968. Homothallism, heterothallism and interspecific hybridization in the genus Phytophthora. Phytopathology 58:1004-1021.

22. Shepherd, C.J. 1975. Phytophthora cinnamomi: An ancient immigrant to Australia. Search. 6:484-490.

23. Smoyer, K. 1940. Avocado tree decline. Calif. Avocado Assoc. Yearbook 50-51.

24. Swofford, D. L. 2002. PAUP* Phylogenetic Analysis Using Parsimony (* and other methods). Version 4.0b10. Sinauer Associates, Inc., Sunderland, MA.

25. Vos, P., Hogers, R., Bleeker, M., Reijans, M., Vandelee, T., Hornes, M., Frijters, A., Pot, J., Peleman, J., Kuiper, M., and Zabeau, M. 1995. AFLPa new technique for DNA-fingerprinting. Nucleic Acids Res. 23:44074414.

26. Wager, V. A. 1942. Phytophthora cinnamomi and wet soil in relation to the dying-back of avocado trees. Hilgardia 14(9):519-532.

27. Zentmyer, G. A. 1952. A substance stimulating sexual reproduction in Phytophthora cinnamomi. Phytopathology 42:24.

28. Zentmyer, G. A. 1976. Distribution of the Al mating type of Phytophthora cinnamomi. Phytopathology 66:701-703.

29. Zentmyer, G. A. 1980. Phytophthora cinnamomi and the Diseases It Causes. American Phytopathological Society, St. Paul, MN.

30. Zentmyer, G. A. 1988. Origin and distribution of four species of Phytophthora. Trans. Br. Mycol. Soc. 91:367-378. 